\title{
Environmental and Motion Effects on Orthogonal Frequency Division Multiplexed On-Off Keying
}

\author{
Paul J. Gendron and T.C. Yang \\ Naval Research Laboratory, 4555 Overlook Avenue SW, Washington DC 20375
}

\begin{abstract}
Orthogonal frequency division multiplexing with on-off keying (OFDM-OOK) offers a means to near BPSK signaling rates with the simplicity of non-coherent processing. At high frequencies source receiver acceleration induces time varying signal dilations that adversely effect both frequency alignment and multicarrier orthogonality. Dilation process estimation is coupled with a decision directed frequency domain channel magnitude response estimator for non-coherent equalization of OFDM-OOK. Joint co-channel estimation is presented for near optimal decisions under loss of orthogonality due to Doppler spreading. Data from a moving source experiment at ranges from $.8 \mathrm{~km}$ to $2.0 \mathrm{~km}$, conducted in the shallow water off the coast of Elba Italy were used to test the feasibility of OFDM-OOK at $18 \mathrm{kHz}$ center frequency with $4 \mathrm{kHz}$ bandwidth. The effects of source receiver relative motion, frequency selectivity, and Doppler spreading on bit error rates were assessed. Mollification of frequency selective fading by diversity combining is demonstrated.
\end{abstract}

\section{INTRODUCTION}

Modulation strategies for underwater communication are typically categorized broadly as coherent and non-coherent. Coherent strategies require the fairly accurate estimation of the channel impulse response to attain relatively high rates $(>1 \mathrm{bps} / \mathrm{Hz}$ ). Diverse phase coherent approaches have been proposed and demonstrated, from phase shift keying (PSK) schemes [1] to multi carrier orthogonal frequency division multiplexing (OFDM) [2]. Single carrier PSK schemes are well suited to computationally fast in-time recursions for channel and Doppler tracking; the LMS and RLS algorithms for channel response estimation and the phase locked loop for Doppler correction are naturally employed in the PSK framework. The equalization of coherent OFDM signals against frequency selective fading is usually aided with training/pilot symbols [3][2] similar to the PSK approach with Doppler estimation critical just as in PSK signaling [4][2].

Low rate non-coherent approaches, such as frequency shift keying (FSK) on the other hand, rely simply on energy detection requiring little or no knowledge of the channel and have demonstrated success in severely Doppler spread channels. In the powerlimited and bandwidth-unconstrained case these simple energy detection approaches attain channel capacity for both time invariant and fading channels [6]. Indeed for this limiting case the attainable rate is $\left(1-2 B_{d} \tau_{\max }\right) P / N_{o}[b p s]$ where $B_{d}$ and $\tau_{\max }$ are the Doppler spread and delay spread of the channel respectively, $P$ is the signal power available and $N_{o}$ the power of the noise process per $\mathrm{Hz}$.

The goal of this paper is to consider OFDM with on-off keying (OFDM-OOK) on 
TABLE 1. MREA03 OFDM-OOK data packet parameters

\begin{tabular}{lcrcrrr}
\hline center freq. & bandwidth & $T_{s}$ & duration & $\tau_{\max }$ & bps & bps/Hz \\
\hline $18 \mathrm{kHz}$ & $4 \mathrm{kHz}$ & $.5 \mathrm{sec}$ & $8 \mathrm{sec}$ & $.125 \mathrm{sec}$ & $3.2 \mathrm{kbps}$ & .8 \\
\hline
\end{tabular}

each tightly spaced (i.e. $\Delta f=1 / T_{s}$ ) subcarrier as a noncoherent scheme for higher rates than are typically expected from energy detection strategies. OFDM is ideally suited for time-dispersive channels as typically encountered in RF communications, however for frequency dispersive underwater accoustic channels with accelerating sources significant interference between subcarriers leads to bit errors as decision variables become dependent across neighboring subcarriers [5]. Frequency selective fading poses constraints on OFDM systems requiring the use of time-frequency interleaving with error correcting coding to attain frequency and time diversity. It is demonstrated with at sea data that uncoded bit error rates are largely associated with channel frequency bands exhibiting low spectral levels.

A sub-optimal receiver processor is used to test the feasibility of OFDM-OOK. Doppler and symbol timing estimation is performed via a spline model of the time varying dilation process. Channel magnitude response measurement is by decision directed estimation without pilot symbols. Symbol decision rules that incorporate Doppler induced dependencies across neighboring sub-carriers is presented to minimize the error rate due to intercarrier interference. Since OFDM is particularly susceptible to motion induced Doppler the scheme is tested from an accelerating source to demonstrate the feasibility of the approach.

\section{MREA03 Experiment}

An OFDM acoustic communication experiment was conducted during MREA03 on June 202003 off the north coast of Elba Italy in 87m of water. The sound speed profile is downward refracting and repeated interaction with the soft bottom result in a multipath delay spread of $\tau_{\max }=.125 \mathrm{sec}$ and an assumed coherence time of $T_{c}>T_{s}=.5 \mathrm{sec}$. From these constraints OFDM-OOK communication packets of 8 seconds duration consisting of $\approx 24$ kbits per packet as shown in Table 1 were tested. With the receiver array moored at a mean depth of $31 \mathrm{~m}$, the source was towed from behind a ship at an approximate depth of $25 \mathrm{~m}$ with speed increasing to 4 knots.

Figure 1a) depicts the Doppler shift measured over the duration of the 8 OFDM-OOK signals at ranges from $.8 \mathrm{~km}$ to $2.0 \mathrm{~km}$. The source track includes both cross range, azimuthally accelerating (first packet), outbound accelerating (second and third packets) as well as constant range rate packets. All of the packets have associated intra-packet time varying Doppler as depicted in Figure 1b) for the fourth packet. 
a)

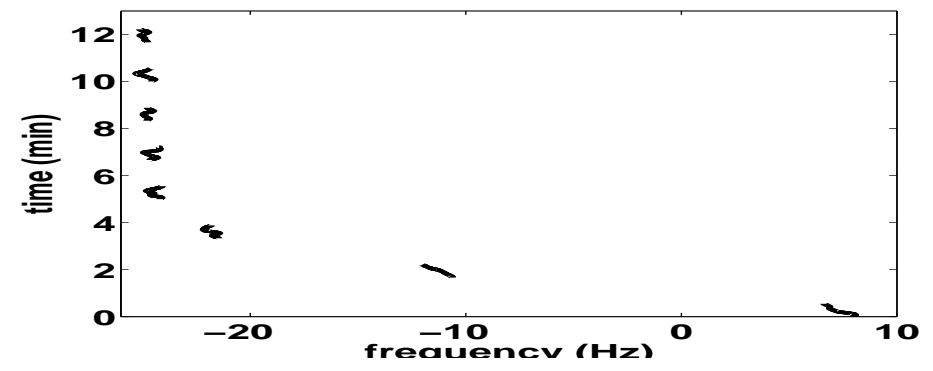

b)

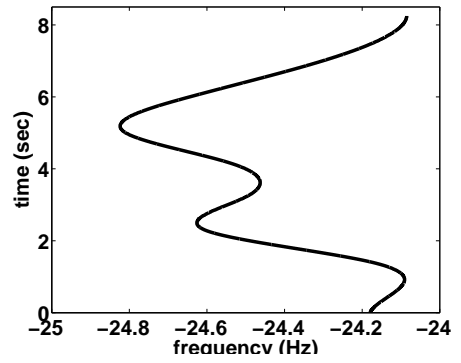

FIGURE 1. a) Doppler associated with the 18kHz MREA03 OFDM-OOK experiment on June 202003. Experiment starts at .8km with negative range rate accelerating to $2 \mathrm{~m} / \mathrm{s}$ for the remainder of experiment (time scale of packets are extended for display). b) To the right, intra-packet Doppler at 5 minutes.

\section{OFDM-OOK SIGNALING MODEL}

Under the assumption that $\tau_{\max }$ is known the source signal consists of a sequence of $L$ symboling intervals of duration $T_{s}$ seperated by $\tau_{\max }$. Let $\alpha_{l}(t)$ represent the dilation process at the source over the $l^{\text {th }}$ signaling interval. The $l^{\text {th }}$ dilated source symbol $s_{\alpha, l}(t)$ is a superpostion of $T_{s}^{\prime}=\int_{(l-1) T_{o}}^{l T_{o}-\tau_{\max }} \alpha^{\prime}(t) d t$ duration dilated tones spaced $\approx 1 / T_{s}^{\prime} \mathrm{Hz}$ apart.

$$
s_{\alpha, l}(t)=A \sqrt{\frac{d \alpha}{d t}} \sum_{k=-K}^{K-1} c_{k, l} g\left(\alpha\left(t-l T_{o}\right)\right) e^{i 2 \pi f_{k} \alpha(t)+\phi_{k}} \quad c_{k, l} \in\{0,1\}
$$

where $f_{k}=f_{c}+k / T_{s}$, is the $k^{t h}$ subcarrier and $T_{o}=T_{s}+\tau_{\text {max }}$. With bandwidth $B$ there are $2 K=B T_{s}$ such tones per symboling interval. The windowing function $g\left(t-l T_{o}\right)=1$ for $(l-1)\left(T_{o}\right)<t<l T_{o}$ and 0 otherwise. The phases $\phi_{k}$ are uncorrelated and serve to minimize the peak to average signal level. The channel symbols, c, encode the information bits $\mathbf{b}, b_{n} n=1, \ldots 2 R K L$ via the $R=1 / 2$ rate convolutional code with a time-frequency interleaver as $\mathbf{c}=C \mathbf{b}$ where $C$ represents both the encoding and interleaving operator.

At depth $d$ the contribution from $s_{\alpha, l}(t)$ at the receiver is $r_{d, l}(t)=\int h_{d, l}(\tau) s_{\alpha, l}(t-$ $\tau) d \tau+e(d, t)$ where $e(d, t)$ is the ambient noise at depth $d$ with spatial coherence $\Sigma$ and is uncorrelated in time due to a prewhitening filter of insignificant duration relative to $h_{d, l}(\tau)$. Figure 2 depicts the noise process spatial coherence at $30 \mathrm{~m}$ depth for a $1.8 \mathrm{~m}$ aperature over a 12 minute interval.

Doppler spread associated with source acceleration. To isolate the effect of the time-varying source dilation on the received OFDM signal let the dilation process for the moving source be described by a quadratc spline of sufficient knots. For the $l^{\text {th }}$ symbol interval $t^{\prime}=\alpha_{l}(t)=\alpha_{0, l}+\alpha_{1, l} t+\alpha_{2, l} t^{2}$ where $\alpha_{1, l} \approx 1-v / c$ and $\alpha_{2, l} \approx a / c$ is proportional to the acceleration of the source divided by the average sound speed at the source such that acceleration towards (away) the receiver implies $\alpha_{2, l}>0\left(\alpha_{2, l}<\right.$ 0 ). From two pilot tones the dilation process is estimated by fitting a spline to the 

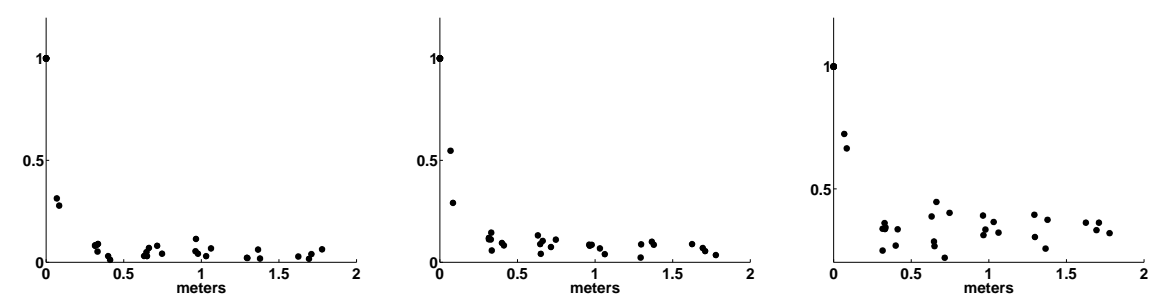

FIGURE 2. Spatial coherence of noise at vertical receiver array on June 20, 2003, at start of experiment (left) at 6 minutes (center) and at 12 minutes (right)

instantaneous frequency $d \alpha(t) / d t=f(t) / f_{c}$. A high resolution Fourier method is used for this purpose. The method is made computationally efficient with the chirp transform algorithm. The resulting expectation is weighted least squares with variances at each sample of the frequency spectrum approximated by the inverse Hessian [7]. Denote these spline parameters by $\hat{\alpha}_{l}(t)$. Figure 3 displays the source motion induced time varying Doppler for a single tone at one phone and compares the residual Doppler spread averaged over two tones and all 6 phones associated with the spline model of Doppler and a constant Doppler model. The spline model effectively demodulates the received signal. To assess the effect of source motion on the modulation strategy at high signal-
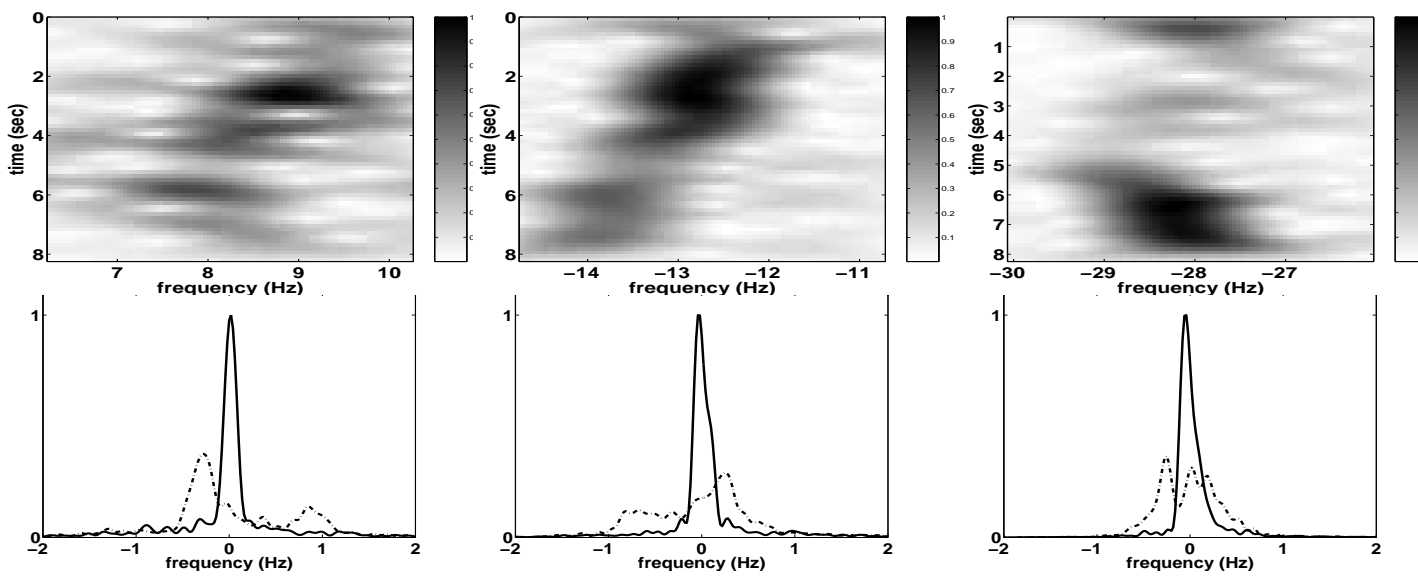

FIGURE 3. Time varying Doppler of $20.429 \mathrm{kHz}$ tone at phone \# 1 and averaged spectra of over all 6 phones with Doppler mean compensation (dashed) and spline based Doppler compensation (solid).

to-noise ratio assume perfect estimation of the dilation process $\hat{\alpha}_{l}(t)=\alpha_{l}(t)$. Consider the receiver contribution at depth $d$, letting source dilated time $t^{\prime}=\alpha_{l}(t)$ with inverse $t=\zeta_{l}\left(t^{\prime}\right)$ it follows that

$$
\begin{array}{r}
r\left(d, \zeta_{l}\left(t^{\prime}\right), l\right) \approx A \sqrt{\frac{d \alpha_{l}}{d t}} \sum_{k} c_{k, l} e^{j 2 \pi f_{k} t^{\prime}} \int_{0}^{\tau_{\max }} \breve{h}(\tau, l) e^{-j 2 \pi f_{k} \alpha_{1, l} \tau} g\left(\alpha\left(t-l T_{o}-\tau\right)\right) \times \\
e^{-j 2 \pi f_{k} \alpha_{2, l} \zeta_{1, l} t^{\prime} \tau} d \tau+e\left(t^{\prime}\right)
\end{array}
$$


where $\breve{h}(d, \tau, l)=h(d, \tau, l) e^{j \pi f_{k}\left(\alpha_{2, l} \zeta_{0, l}+\alpha_{2, l} \tau^{2}\right)}$. Assume that $\alpha_{2, l} \zeta_{2, l}<<1$ and it follows that the dilated received signal $r_{\zeta}\left(d, t^{\prime}, l\right)=\sqrt{d \zeta_{l} / d t^{\prime}} r\left(d, \zeta_{l}\left(t^{\prime}\right), l\right)$ yields the finite duration Fourier transform

$$
\begin{array}{r}
\tilde{r}(d, f, l)=\int_{(l-1) T_{o}+\tau_{\max }}^{l T_{o}} r_{\zeta_{l}}\left(d, t^{\prime}, l\right) e^{-j 2 \pi f t^{\prime}} d t^{\prime}= \\
A T_{s} \sum_{k} c_{k, l} \int_{0}^{\tau_{\max }} \check{h}(d, \tau, l) e^{-j 2 \pi f_{k} \alpha_{1, l} \tau} \operatorname{sinc}\left(T_{s}\left(f_{k}\left(1-\alpha_{2, l} \zeta_{1, l} \tau\right)-f\right)\right) d \tau+\tilde{e}(f) .
\end{array}
$$

Equation 2 is a superpostion (in $\tau$ ) of sinc kernals (each frequency shifted by $\approx \alpha_{2, l} \tau$ ) and weighted by the chirp modulated impulse response. For constant velocity motion $\alpha_{2, l}=0$ and the time invariant assumption over the symboling interval $T_{s}$, it follows that $r(f, l)=T_{s} \sum_{k} c_{k, l} \tilde{h}\left(f_{k} \alpha_{1, l}, l\right) \operatorname{sinc}\left(T_{s}\left(f_{k}-f\right)\right)$; no ICI at the receiver. For sources radially accelerating towards the receiver, energy from $c_{k, l}$ in the received demodulated signal is roughly spread to lower frequencies $f_{k}\left(1-\alpha_{2, l} \tau_{\max }\right)<f<f_{k}$. Likewise for a source accelerating away from the receiver, $\alpha_{2, l}<0$ and spreading of the source tone at $f_{k}$ is skewed to the frequency band $f_{k}<f<f_{k}\left(1-\alpha_{2, l} \tau_{\max }\right)$. It is implied then that with conventional processing (i.e. demodulate, dilate and integrate) of OFDM signals the channel is effectively time variant even over durations as short as the symbol interval $T_{s}$. Expand $\operatorname{sinc}\left(T_{s}\left(f_{k}\left(1-\alpha_{2, l} \zeta_{1, l} \tau\right)-f\right)=\sum_{m} \gamma_{m-k}(\tau) \operatorname{sinc}\left(T_{s}\left(f_{k}-f-f_{m}\right)\right)\right.$ where $\left|\gamma_{m}\right|^{2} \propto 1 / m^{2}$ to explicitly display the loss of orthogonality. In short, radial acceleration imparts a spread that is roughly proportional to the product of acceleration and channel delay spread. Each channel coefficient $c_{k, l}$ associated with frequency $f_{k}$ and time $l T_{o}$ contributes to the frequency slots of its neighbors even under time invariant channel conditions. In addition differential Doppler associated with different dilations along individual ray paths lead to further Doppler spreading for source environment scenarios where eigenrays exhibit signficant launching angles.

Detection. With on-off keying, non-coherent detection is employed at the receiver with the ICI model truncated to neighboring frequencies. The resulting model of the received signal at the $D$ phones is $\tilde{\mathbf{r}}(k, l) \mid H(k, l), \mathbf{c}_{k}^{l}, \Sigma \sim \mathscr{N}\left(H(k, l) \mathbf{c}_{k}^{l}, \Sigma\right)$ where $H(k, l)$ represents the $C^{D x 3}$ channel transfer function from source frequencies $f_{k-1} . . f_{k+1}$ to received frequency $f_{k}$ at symboling instant $l, \mathbf{c}_{k}^{l}=\left[c_{k-1, l}, c_{k, l}, c_{k+1, l}\right]^{\prime} . \Sigma$, assumed known, is the spatial covariance of the noise. This assumption is reasonable for high SNR in cases where the receiver has time-bandwidth sufficient to accurately estimate $\Sigma$. With a view towards on-off keying (i.e. $c_{k}^{2}=c_{k}$ ) this truncated ICI approximation implies that $\rho_{k, l}=\tilde{\mathbf{r}}^{\prime}(k, l) \Sigma^{-1} \tilde{\mathbf{r}}(k, l)$ is distributed as [8] [9] $p\left(\rho_{k, l} \mid H(k, l), \mathbf{c}_{k}^{l}, \Sigma\right)=$ $\chi_{2 D}^{2}\left(\mathbf{c}_{k}^{\prime} H(k, l)^{\prime} \Sigma^{-1} H(k, l) \mathbf{c}_{k}^{l}\right)$ where $\chi_{2 D}^{2}\left(\lambda^{2}\right)$ is a non-central $\chi^{2}$ distribution with noncentrality parameter $\lambda^{2}$. Approximate the noncentrality parameter, ignoring cross terms as $c_{k-1, k, k+1}^{\prime} H(k, l)^{\prime} \Sigma^{-1} H(k, l) c_{k-1, k, k+1} \approx \sum_{d} \lambda_{k-1, k, l}^{2} c_{k-1, l}+\lambda_{k, k, l}^{2} c_{k, l}+\lambda_{k+1, k, l}^{2} c_{k+1, l}$ where $\lambda_{k-1, k}^{2}=\sum_{d}\left|H_{d, k-1}(k, l)\right|^{2} / \sigma_{d, l}$. For scenarios where the channel is time invariant over the symboling interval and the source is not accelerating there is no ICI and only $\lambda_{k, k, l}^{2}$ persists. The channel gains $\lambda_{k \pm 1, k, l}^{2}$ are approximated with $\lambda_{k \pm 1, k, l}^{2}=\delta_{ \pm} \lambda_{k, k, l}^{2}$ where the factors $\delta_{ \pm}$are empirical Doppler spread estimates from the out of band tones depicted in Figure 3. 
To estimate data in the presense of ICI, iterate over the conditional expectations of each of the unknown parameters; data, $\mathbf{c}_{l}$, and channel gains, $\Lambda_{l}$, without consideration of dependencies associated with the convolutional code. The approach is warranted for its computational efficiency; with interleaving over time it is necessary to estimate the source data $c_{k, l}$ without Viterbi decoding so that estimation of the channel transfer function can be accomplished. Final estimation of data and channel can be made via the Viterbi decoder once the entire channel response is initally estimated. With this in mind start with the conditional expectation of the channel bits given adjacent bits and the channel magnitude response

$$
\begin{array}{r}
\hat{c}_{k, l}=E\left[c_{k, l} \mid c_{k-1, l}, c_{k+1, l}, \Lambda_{k, l}, \Sigma, \rho(k, l)\right] \approx \\
\frac{\chi_{2 D}^{2}\left(\lambda_{k-1, k, l}^{2} \hat{c}_{k-1, l}+\lambda_{k, k, l}^{2}+\lambda_{k+1, k, l}^{2} \hat{c}_{k+1, l}\right)}{\chi_{2 D}^{2}\left(\lambda_{k-1, k, l}^{2} \hat{c}_{k-1, l}+\lambda_{k+1, k, l}^{2} \hat{c}_{k+1, l}\right)+\chi_{2 D}^{2}\left(\lambda_{k-1, k, l}^{2} \hat{c}_{k-1, l}+\lambda_{k, k, l}^{2}+\lambda_{k+1, k, l}^{2} \hat{c}_{k+1, l}\right)} .
\end{array}
$$

Initialing with $\hat{\mathbf{c}}_{l}=0$, two iterations are sufficient for convergence.

Channel Estimation. Estimation of the channel magnitude $E\left[|H|_{l}^{2} \mid \mathbf{r}_{l, \ldots 1}, \hat{\mathbf{c}}_{l, \ldots 1}\right]$ is made by use of an RLS-like recursion from estimates $E\left[|H|_{l-1}^{2} \mid \mathbf{r}_{l-1, \ldots 1}, \hat{\mathbf{c}}_{l-1, \ldots 1}\right]$ and $E\left[\mathbf{c}_{l} \mid \mathbf{r}, E\left[|H|_{l-1}^{2}\right]\right]$. Channel estimates in time-frequency slots $k, l$ for which $c_{k, l}=0$ have zero precision implying that the channel estimate relies solely on the previous estimate based on $\hat{c}_{k, l-1 \ldots 1}$. The algorithm uses an empirical Bayes approach twice iterating between estimates of channel and data. This has proved sufficient for convergence. Figure 4 depicts the channel transfer function magnitude, estimated via the proposed method, for ranges from $.8 \mathrm{~km}$ to $2.0 \mathrm{~km}$ demonstrating the frequency selective nature of the channel and the changing striation pattern with range. At closer ranges, Figures 4a), b) and c) there is greater striation and an implied decrease in coherence time. The vertical coherence length is summarized in Figure 5 and gives a measure of the diverisy present for this configuration. Typical vertical coherence lengths for these channels is between 5 and 10 wavelengths.

\section{RESULTS}

This signal model and receiver structure were tested for uncoded bit error rate (BER) performance. Figure 6 lists BERs for 6 different ranges and source motion scenarios with a symetric ICI model $\left(\delta_{ \pm}=\delta\right)$. Since SNR varies over range the received SNR is augmented by adding Gaussian white noise to the packet to control for SNR and compare more effectively performance over different ranges and Doppler motion scenarios. It is evident that the two accelerating source packets yield BERs that are typical of the sets while packets at ranges $1 \mathrm{~km}, 1.6 \mathrm{~km}$, and $2.0 \mathrm{~km}$ which experience the greatest degree of nulling in the channel transfer function have relatively large BERs. This suggests that channel frequency selectivity is a dominant factor for OFDM-OOK performance. 


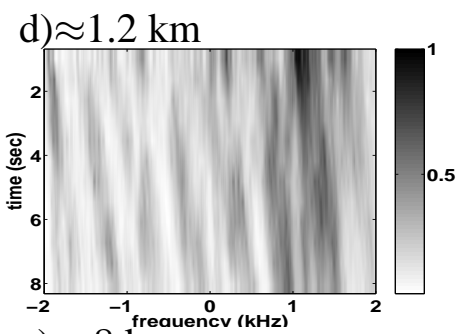

a) $\approx .8 \mathrm{~km}$

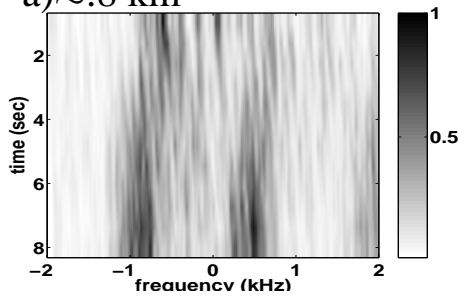

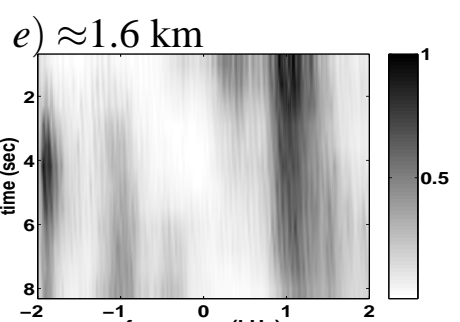

b) $\approx .9 \mathrm{~km}$

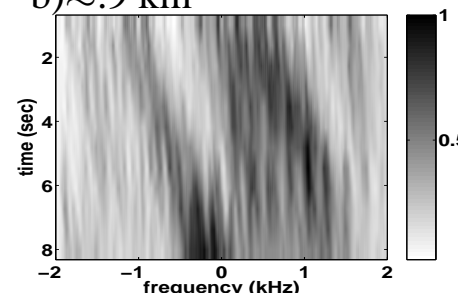

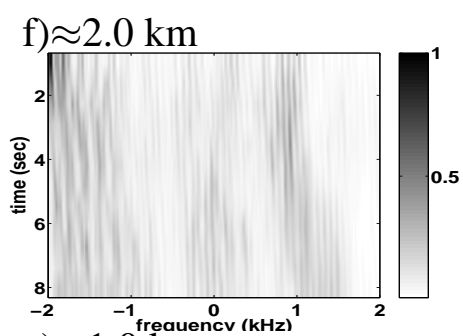

c) $\approx 1.0 \mathrm{~km}$

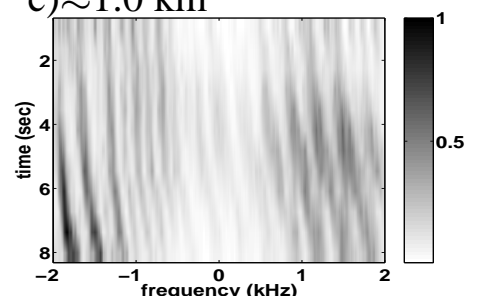

FIGURE 4. Channel transfer function magnitude estimated via decision directed RLS algorithm at ranges from $.8 \mathrm{~km}$ to $2.0 \mathrm{~km}$ showing frequency selective fading.
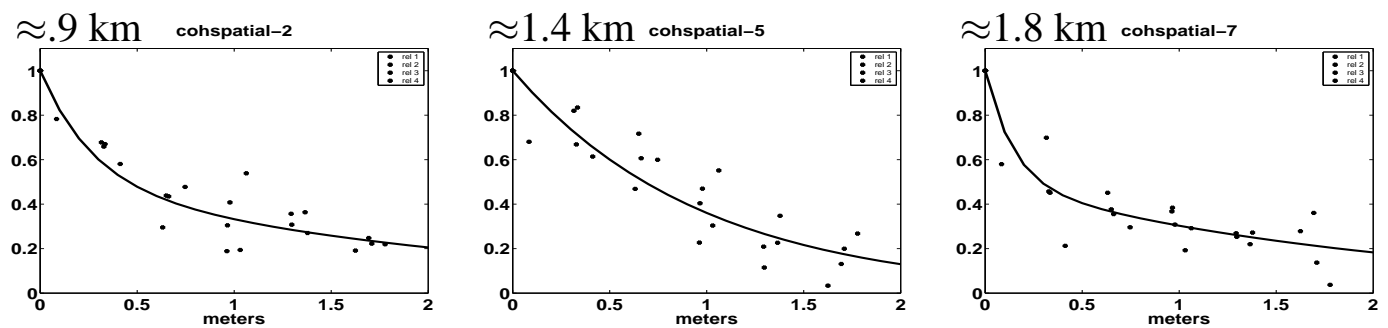

FIGURE 5. Vertical coherence of channel at various ranges over $1.8 \mathrm{~m}$ aperature at receiver depth of $30 \mathrm{~m}$.

\section{SUMMARY AND DISCUSSION}

OFDM with on-off keying is considered as a means of attaining near BPSK rates with non-coherent processing. This paper evaluates OFDM-OOK bit error rates under time varying Doppler conditions (towed source speed at $\approx 4$ knots and non-negligible accelerations) contrasted with previous data limited to small Doppler shifts. Doppler spread estimation is made empirically from out of band tones and gives a measure of the ICI used to remove bias in the estimation of channel bits at each subcarrier. The algorithm employs a decision directed RLS-like channel magnitude estimator to yield stable performance at SNRs below $12 \mathrm{~dB}$. Lastly the utility of modeling skew in Doppler spread is a current topic of interest and its causes such as source radial velocity, acceleration and wind induced surface waves require further study. 

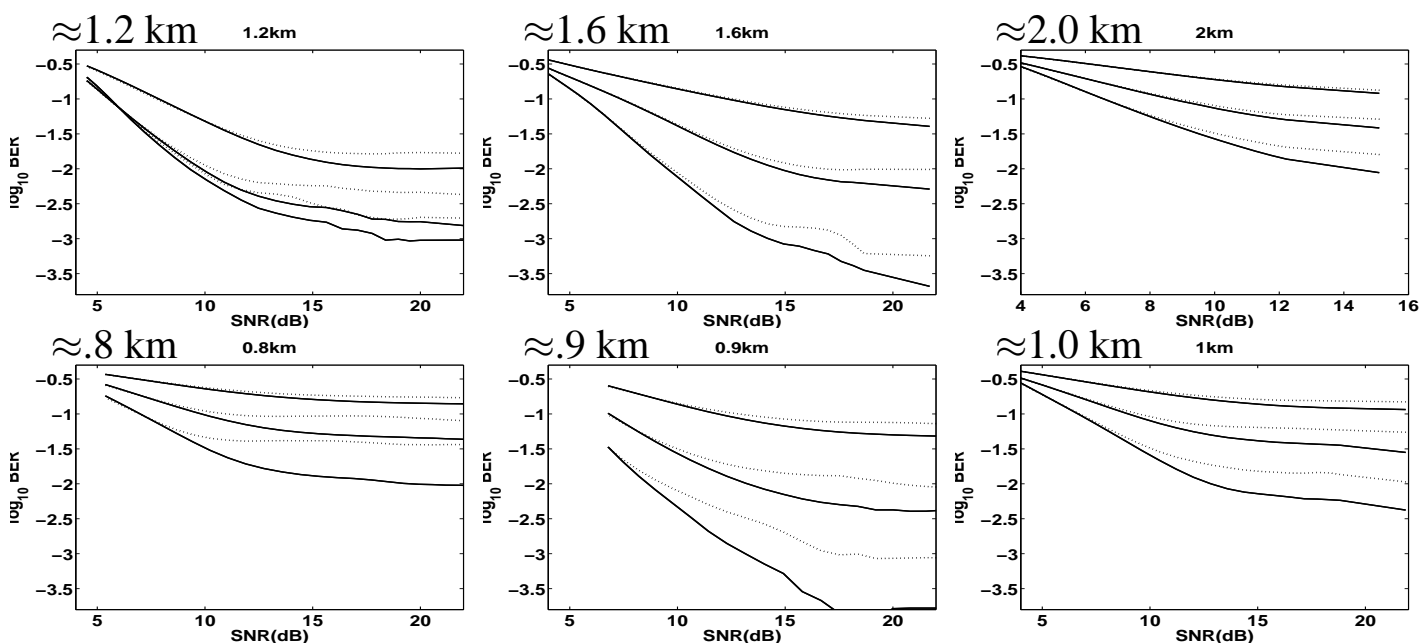

FIGURE 6. Uncoded bit error rates for diversity levels of 1, 3 and 6 at moderate SNRs and ranges from $.8 \mathrm{~km}$ to $2.0 \mathrm{~km}$. Dashed line shows performance without ICI model and with channel assumed perfectly coherent across symboling frames.

\section{ACKNOWLEDGMENTS}

This work supported by the Office of Naval Research. Thanks to NURC for the opportunity to participate in the MREA03 experiment; Jeff Schindall, Mike McCord and Wen-Bin Yang for experiment execution; Bruce Pasewark and Thomas Hayward for helpful discussions.

\section{REFERENCES}

1. Stojanovic, M., Journal of Oceanic Engineering, 21, 125-136 (1996).

2. Kim, B., and Lu, I., Journal of Acoustical Society of America, 109, 2477 (2001).

3. Li, Y., Cimini, L., and Sollenberger, N., IEEE Transactions on Communications, 46, 902-915 (1998).

4. Luise, M., and Reggiannini, R., IEEE Transaction on Communications, 44, 1590-1598 (1996).

5. Davies, J. J., and Pointer, S. A., Oceans '98 Coference Proceedings, 2, 1022-1027 (1998).

6. Telatar, I., and Tse, D., IEEE Transactions on Information Theory, 46, 1384-1400 (2000).

7. Rife, D., and Boorstyn, R., IEEE Transactions on Information Theory, 20, 591-598 (1974).

8. Mäkaläinen, T., Commentationes Physico-Mathematicae, Societas Scientarium Fennica, 31, 1-6 (1966).

9. Johnson, N., and Kotz, S., Continuous Univariate Distributions-2, John Wiley \& Sons, Inc., 605 Third Avenue, New York, NY 10158, 1972, pp. 189-194. 